27

\section{USE OF SOUND-ABSORBING PANEL TO REDUCE NOISY INCUBATOR} REVERBERATING EFFECTS

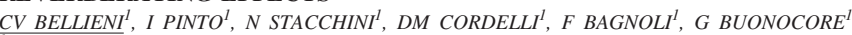
${ }^{T}$ UNIVERSITY OF SIENA (ITALY)

OBJECTIVES: To assess the amplification and distortion of noise in incubators by measuring noise levels from various sources under various conditions.

STUDY DESIGN: We recorded sound pressure levels (SPL) of background noise, baby crying, alarms and porthole closing with the incubator hood open or closed. Then we tried to reduce the sound level in the incubator by applying a sound absorbing panel to the ceiling of the hood.

RESULTS: The sound sources analysed produced very high SPL. In free field baby crying and porthole noise were, respectively, $81-83 \mathrm{~dB}$ and $70-71 \mathrm{~dB}$, closing the hood they raised to $84-87$ an $73-74 \mathrm{~dB}$ : this means that the noise perceived by a baby in the incubator had twice the SPL of the same noise emitted in a free field; alarm noise was $56-57 \mathrm{~dB}$ inside incubators. Sound emitted inside the hood was also distorted, i.e. it had different features from the same source in a free field. The insulating panel prevented amplification and distortion due to the hood and reduced the SPL of alarms. Only background noise did not decrease with the panel. Sound absorbing panel reduced baby's crying, porthole noise and alarm noise SPL to $82-85,70-71$ and $50-51 \mathrm{~dB}$, respectively.

CONCLUSIONS: Incubators are reverberating environments, which amplify the baby's cry and other noises produced inside the hood. The frequency components of noises are altered by the hood. The sound absorbing panel reduced this phenomenon.

\section{8}

BLINK-STARTLE REFLEX HABITUATION IN 30-34 WEEK LOW-RISK FETUSES

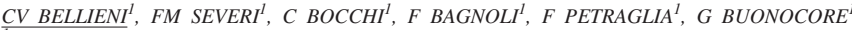

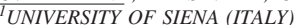

OBJECTIVE: To investigate whether the blink-startle reflex (BSR) is a good marker of habituation to vibroacoustic stimulation in healthy preterm fetuses.

MATERIALS AND METHODS: Twenty-two women with uncomplicated pregnancies at 30-34 weeks participated. The vibroacoustic stimulus was repeatedly applied to the maternal abdomen above the fetal head for a period of $2 \mathrm{~s}$ every $10 \mathrm{~s}$. Fetal eye thickening monitored by US within $2 \mathrm{~s}$ of the stimulus was considered a positive response. The habituation rate was defined as the number of stimul applied before the fetus stopped responding to two consecutive stimuli. Results were compared using the Mann-Whitney U test.

RESULTS: All 22 fetuses showed habituation at a rate that varied from 1 to 9 (mean $4.2 \pm 1.8$ ). CONCLUSION: BSR is a good marker of habituation in preterm fetuses. Further study is needed to ascertain whether abnormal BSR habituation could be a sign of fetal suffering.

\section{9}

\section{FEEDING PRACTICES AMONG TERTIARY-LEVEL NEONATAL UNITS IN NORTH ITALY}

$R$ BELLU ${ }^{\prime}, R$ ZANINI ${ }^{1}, L$ GAGLIARDI ${ }^{2}{ }^{1}$ NICU OSPEDALE MANZONI,${ }^{2}$ PEDIATRIC DEPARTMENT - OSPEDALE UNICO DELLA VERSILIA (ITALY)

Objectives: To describe feeding practice among 13 tertiary-level neonatal as regard to the use of maternal and artificial milk at discharge for very low birth weight infants $(<=1500 \mathrm{~g})$.

Methods. A cohort of 1372 surviving neonates, admitted to 13 tertiary-level neonatal units in Lombardy (Northern Italy) in 1999-2002 with birth weight $<=1500 \mathrm{~g}$, was studied using data collected for the Italian Neonatal Network. Feeding practices were routinelly recorded in the Network as the use at discharge of maternal milk (fortified or not), artificial milk or both. Analysis were performed by contingency tables and by modelling differences among centers with logistic regression including covariates such as birth weight, gestational age, mode of delivery 5 min Apgar score, CRIB score, place of delivery, sex of the newborn, year of birth.

Results. The percentage of neonates discharged on maternal milk and artificial milk ranged between 4,6 to $52,3 \%$ and 12,5 to $85,4 \%$ respectivelly among centers. Neonates discharged on maternal milk had higher birth weight, gestational age and 5 min Apgar score. Nevertheless, wide differences were observed between centers in multivariate regression models including birth weight, gestational age, 5 min Apgar score, location of birth (inborn versus outborn), mode of delivery and CRIB score.

Conclusions. These results support the hypothesis that differences in feeding practice at discharge are much more center-dependent, even in a uniform and high standard of care area, than dependent on individual characteristics of the neonates. This point to the problem of a wide variance in practice that deserves further investigation.
30

MEDICAL INJURIES AND CONGENITAL HEART SURGERY

OJ BENAVIDEZI, K GAUVREAU ${ }^{l}$, KJ JENKINS ${ }^{l}$ CHILDRENS HOSPITAL BOSTON, HARVARD MEDICAL SCHOOL (USA)

BACKGROUND Complex pediatric patients have been identified as being a vulnerable group for medical injury. Comprehensive assessment of the prevalence of injury events among children undergoing congenital heart surgery has not been performed.

OBJECTIVE We examined the 1) prevalence of medical injury codes and 2) the relationship of reported medical injury codes to mortality among pediatric cases of congenital heart surgery.

DESIGN/METHOD Data were obtained from the Healthcare Cost and Utilization Project Kids Inpatient Database 2000. We identified inpatient discharges of cases $<18$ years of age who underwent congenital heart surgery using ICD-9-CM codes. A validated algorithm was used to identify codes indicating medical injury. Medical injury codes were categorized as related to: 1) procedure, 2) device, implant or graft, 3) drug or biologic, or 4) radiation.

RESULTS Among the 12,717 cardiac surgical cases identified, 4014 (32\%) had at least one medical injury code; there were a total of 6650 codes of medical injury ( 523 injuries per 1000 cases). Procedure related injury codes represented $75 \%$ of reported injury codes (392 per 1000 cases); device, implant, or graft related injuries represented $21 \%$, (111 per 1000 cases); drug/biologic related injuries represented $4 \%$, (20 per 1000 cases) there were no radiation injury codes identified.

Among the procedure related injury codes, respiratory complications represented $18 \%$ of all injury codes (94/1000 cases); cardiac complications represented 17\%, (87/1000 cases); Hematoma/hemorrhage complications $9 \%,(48 / 1000$ cases); vascular complications $7 \%,(36 / 1000$ cases); and infectious complications $4 \%$ of all injury codes (20/1000cases). Patients who died had a greater rate of medical errors $(505 / 1000$ cases vs. $306 / 1000$ cases, $\mathrm{p}<0.001)$.

CONCLUSION Medical injury codes are frequently reported among congenital heart surgery case and are associated with mortality. The most common type of medical injury is procedure related. Additional work is needed to identify patient factors predictive of medical injuries.

\section{1}

ASSOCIATION OF MEDICAL INJURY AND MORTALITY DURING CONGENITAL HEART SURGERY ADMISSIONS

OJ BENAVIDEZ ${ }^{1}, K$ GAUVREAU ${ }^{1}$, KJ JENKINS ${ }^{1}{ }^{1}$ CHILDRENS HOSPTIAL BOSTON, HARVARD MEDICAL SCHOOL (USA)

Background Despite complexity of care and high death risk, there has been no broad assessment of medical injury rates or its association with mortality following congenital heart surgery. Our objectives were to examine the rate of medical injuries and he independent effect of medical injuries on risk-adjusted mortality during congenital heart surgery admissions.

Methods We identified congenital heart surgery admissions, ages $<18$ years, within the Kids Inpatient Database 2000, and applied a previously validated medical injury screening method using ICD-9-CM codes. Medical injuries were classified into four categories: 1) drugs; 2) procedures; 3) devices, implants, and grafts; and 4) radiation related. Adjustment for case-mix was performed using the Risk Adjustment in Congenital Heart Surgery method. We examined the independent effect of a medical injury code and medical injury subcategory on risk-adjusted mortality using generalized estimating equations controlling for case-mix and reported predictors for mortality.

Results Among the 10,032 congenital heart surgical admissions identified, 5242 medical injury codes were present at a rate of 523 injuries per 1000 discharges. Injury codes were procedure related $78 \%, 408$ per 1000 discharges; device, implant, or graft related $18 \%, 96$ per 1000; drug related $4 \%$, 19 per 1000; no radiation injury codes were identified. After adjusting for case-mix, gender, race, insurance and surgical volume cases with medical injury codes had a substantially greater odds of death compared to cases without medical injury codes, OR $2.4(\mathrm{p}<0.001)$. Medical injury subcategories: procedure OR 2.3, $\mathrm{p}<0.001$; device, implant, or graft OR $2.7(\mathrm{p}<0.001)$ related injuries; drug related injuries OR $0.7(\mathrm{p}=\mathrm{NS})$.

Conclusions Medical injuries are common during admissions for congenital heart surgery and are associated with an increased risk of death even after controlling for known predictors for death. Strategies to reduce medical injury might reduce death in this complex pediatric population.
GBS PREVENTION POLICIES IN A NORTH ITALIAN AREA

A BERARDI I LUGLI K ROSSI I E DELLA CASA MATTINI I C GALLO ${ }^{l}$ G GARGANO ${ }^{l}$ E TRIDAPALLI ${ }^{2}$, M STELLA ${ }^{2}, F$ FERRARI ${ }^{I}$, EMILIA-ROMAGNA GBS PREV. WORKING GROUP ${ }^{l}$ ${ }^{I}$ N.I.C.C.U. BOLOGNA (OSPEDALE SANT'ORSOLA), ${ }^{2}$ N.I.C.U. MODENA (ITALY)

Background Group B streptococcus (GBS) is a leading cause of neonatal early-onset infections (EOI). In USA infections decreased after adoption of prevention guidelines, while in Europe strategies are low-developed. We retrospectively investigated prevention strategies in an Italian area (35000 live birth/year)

Method Twenty-eight birth-centres of Emilia-Romagna were asked to fulfil a questionnaire about prevention strategies adopted during the 2000 and 2003. We promoted periodical meetings to develop and share prevention guidelines. Management of pregnant women and newborns were analyzed.

Results: Fifteen and 27 centres participated to the study during 2000 and 2003 respectively. All centres used a screening-based approach. Eight $(57,1 \%)$ in 2000 and $19(70.4 \%)$ centres in 2003 collected maternal swabs at the lower third of vagina; rectal cultures or selective broth media were not used in 2000. Fourteen centres (51.9\%) used vagino-rectal swabs and $5(18.5 \%)$ selective broth media in 2003. If intrapartum chemoprophylaxis (IAP) was inadequate, thirteen centres $(92.8 \%$ ) in 2000 screened neonatal colonization, most of them $(78.6 \%)$ treating colonized infants with antibiotics. In 2003 a few centres assessed colonization (37\%) and treated (29.6\%) infants. In 2000 only two centres (14.3\%) collected blood cultures in 'at risk' newborn, while sixteen centres (59.2\%) did in 2003. Changes were more prominent in centres participating since 2000.

Conclusion Knowledge of guidelines among professionals and health-care providers is a good too to obtain changes in GBS policies. Screening of pregnant women and ascertainment of neonatal infection significantly improved, avoiding newborn over treatment. 